Behçet's disease (NB) and can be classified into acute type (ANB) and chronic progressive type (CPNB) based upon differences in the clinical course and responses to corticosteroid treatment. Diagnostic criteria were generated in 2013 based on a multicenter clinical survey performed by the Behçet's Disease Research Committee of the Ministry of Health, Labor and Welfare of the Japanese Government. Although "Guidelines for Treatment of NB" was also proposed based on the survey, it is still preliminary.

Objectives: The aim of the current study is to develop evidence-based recommendations for the management of NB supplemented by expert opinions where necessary.

Methods: First, clinical questions (CQs) on NB were extracted from a literature search for problem areas and related keywords, and draft CQs and a flow chart were prepared. The expert committee, a task force of the research subcommittee for NB, consisted of 7 board-certified rheumatologists (one was also a boardcertified neurologist) and 3 board-certified neurologists. A systematic literature search was performed using Medline and the Japan Medical Abstract Society databases from 1997 to 2016. A total of 15 initial CQs were generated. These yielded the final recommendations developed from 3 blind Delphi rounds, in which the rate of agreement scores on CQs (range 1 [disagree]-5 [strongly agree]) was determined though voting by the whole committee.

Results: Thirteen recommendations were developed for the management of NB (general 1, ANB 7, CPNB 5). The strength of each recommendation was established based on the evidence level as well as rate of agreement. There was excellent concordance between the level of agreement of rheumatologists and that of neurologists. Based on these recommendations, a flow chart was established for the management for ANB and CPNB (Figure).

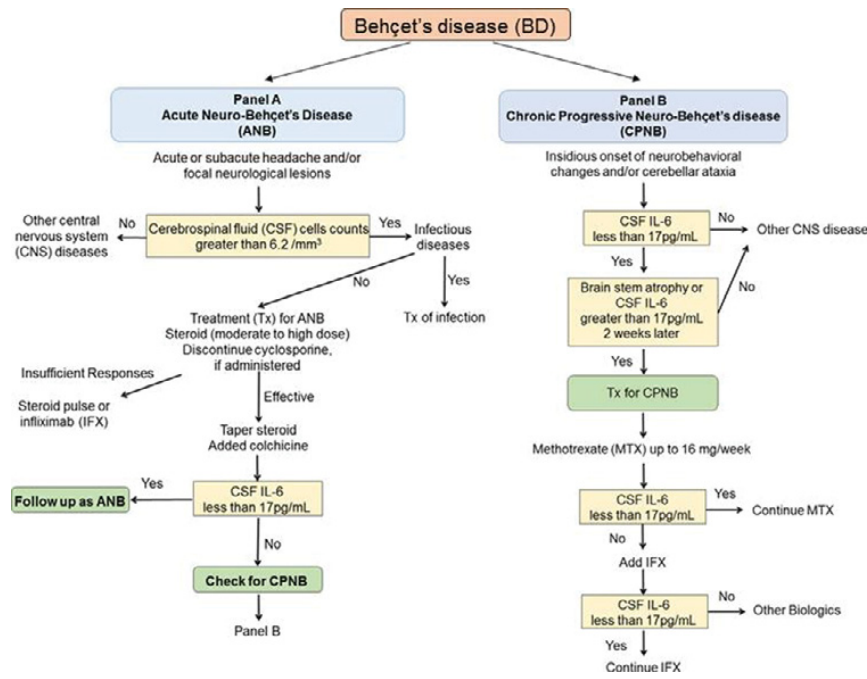

Conclusions: The recommendations generated in this study are mainly based not only on expert opinions but on the results of uncontrolled evidence from open trials and retrospective cohort studies. Guidelines that can be used for international studies are needed, for which verification by further properly designed controlled clinical trials is required.

Disclosure of Interest: None declared

DOI: 10.1136/annrheumdis-2017-eular.1201

\section{FRI0327 GASTROSCOPIC FEATURES AND CLINICAL CHARACTERISTICS IN 172 CASES OF CHILDREN WITH HENOCH-SCHONLEINPURPURA}

H. Zeng. Department of Pediatric Allergy, Immunology and Rheumatolog, Guangzhou Women and Children's Medical Center, Guangzhou, China

Objectives: To investigate gastroscopic features and explore the relationship between clinical characteristics in children with Henoch-Schonleinpuepura (HSP). Methods: To take gastroscope in 172 cases of children with HSP in our hospital and summarize the gastroscopic performance.All the case were divided into two groups by gastroduodenal mucosal bleeding or not.It was compared among the total time of abdominal pain, pain relief, hospitalization, fasting and kidney injury case in the groups.

Results: Gastroscope with varying degrees of injury of 172 cases has accounted for 169 cases (98.3\%).Gastroscopic mainly revealedgastroduodenal mucosal congestion, edema, rough, erosion, bleeding and ulcer, which involved 148 cases of gastric $(86.0 \%), 158$ cases of duodenal involvement $(91.9 \%)$. Mucosal erosion and bleeding occurs mainly in duodenum, mostly in the descending duodenum. Duodenal bleeding accounted for 36 cases $(21.8 \%)$ in the bulb and 92 cases $(55.8 \%)$ in the descendant. Only five cases $(2.9 \%)$ of ulcer occurred in the duodenum, where four cases of bulbar ulcer, one case of descending ulcer. Esophageal and gastric cardia mucosal just occurred in one case. There were not significant difference $(P>0.05)$ among the time of abdominal pain, pain relief, hospitalization and fasting in the group. There was no significant difference
$(P>0.05)$ in the incidence of kidney injury between two groups of children during hospitalization.

Conclusions: Gastroscopic features of HSP in children is characterized by bleeding, erosion of duodenal mucosa and occasional duodenal ulcer formation, which mostly involve the antral mucosa, rarely involving the esophagus, cardia. There was no significant difference $(P>0.05)$ among the severity of gastroscopic performance and the time of abdominal pain, fasting,hospitalization and kidney injury of the cases during hospitalization.

Disclosure of Interest: None declared

DOI: 10.1136/annrheumdis-2017-eular.2127

\section{FRI0328 THE DIAGNOSTIC VALUE OF ALPHA-1-ANTITRYPSIN PHENOTYPE IN SYSTEMIC VASCULITIS}

I. Belyaeva ${ }^{1}$, A. Chudinov ${ }^{2}$, M. Pervakova ${ }^{3}$, V. Mazurov ${ }^{1}$, S. Lapin ${ }^{3}$, O. Inamova ${ }^{2} .{ }^{1}$ North-West State Medical University named after I.I. Mechnikov, Saint-Petersburg, Russia; ${ }^{2}$ Clinical Rheumatological Hospital \#25,

Saint-Petersburg, RUSSIA; ${ }^{3}$ First Pavlov State Medical University of St. Petersburg, Saint-Petersburg, Russian Federation

Background: Deficiency of alpha-1 protease inhibitor, or alpha-1-antitrypsin (A1AT) is a frequent genetic disorder, which is characterized by low serum level of A1AT and usually manifests as pulmonary emphysema and liver disease. Also the deficiency of A1AT is known to be associated with granulomatosis with polyangiitis (GPA). The influence of A1AT deficiency on GPA clinical course is not clarified.

Objectives: The aim of this study was to estimate the prevalence of pathological A1 AT phenotypes in GPA and other systemic vasculitis and to define the influence of A1AT phenotype on clinical course of GPA.

Methods: We enrolled 86 patients with systemic vasculitis, including GPA ( $N=47)$, microscopic polyangiitis (MPA, N=16), eosinophilic granulomatosis with polyangiitis (EGPA, $\mathrm{N}=12$ ), polyarteriitisnodosa ( $\mathrm{PN}, \mathrm{N}=11)$. 46 healthy donors were included in the control group. All blood samples underwent A1AT phenotyping by isoelectrofocusing (IEF) and turbidimetric A1AT measurement. The results of phenotyping were compared to clinical data, such as BVAS activity rate (Birmingham Vasculitis Activity Score), VDI index (vasculitis damage index), organs involvement, inflammatory markers, including antineutrophil cytoplasmic antibodies (ANCA), total IgG concentration and serum levels of C3, C4 complement factors.

Results: Pathological A1AT phenotypes were found in 17\% (8/47) of GPA patients, $6,25 \%(1 / 16)$ of MPA patients, $2 \%(1 / 46)$ of healthy donors and were never found in EGPA, PN. The abnormal phenotypes in GPA were 1PiZZ, 4PiMZ, 2PiMF, 1 PiMS, and 1PiMS phenotype was identified in MPA patient. Lesion of lung and upper respiratory tract was observed in all patients with pathological phenotypes A1AT $(N=8)$, while in normal phenotype A1AT it was present in $72 \%$ and $82 \%$ respectively. The mean concentration of A1AT was significantly lower in GPA patients with abnormal A1AT phenotypes, than in patients with normal phenotype A1AT (respectively $1003 \pm 148.8$ and $1964 \pm 127.9 \mathrm{mg} / \mathrm{L}, p<0,01$ ). The average activity by BVAS index in GPA was significantly higher in patients with pathological phenotype A1AT than in patients with normal phenotype A1AT $(24,63 \pm 2,897$ and $18,05 \pm 1,444$ points, $p<0,05$ ). Also we revealed excess levels of VDI in cohort of patients with abnormal phenotype A1AT rather, than in cohort of patients with normal phenotype A1AT $(6,3 \pm 3,1$ versus $5,4 \pm 2,6, p<0,05)$. The average values concentration of antibodies to proteinase-3 in GPA patients with abnormal phenotype A1AT were significantly higher compared to GPA patients with normal phenotype A1AT (respectively $142,4 \pm 25,24$ and $86,784 \pm 14,98 \mathrm{RU} / \mathrm{ml}, \mathrm{p}<0,05$ ). In GPA patients with mutated A1AT phenotypes levels of serum creatinine concentrations $(p<0,01)$, levels of total lg $G$ concentration and serum levels of $C 3$ and $\mathrm{C} 4$ complement factors $(p<0.05)$ also were significantly higher than in group of GPA patients with normal A1AT phenotype.

Conclusions: Pathological A1AT phenotypes are more often observed in GPA patients who have more severe GPA clinical course and higher immunological disease activity.

Disclosure of Interest: None declared

DOI: 10.1136/annrheumdis-2017-eular.4412

\section{FRI0329 EFFICACY AND SAFETY OF INFLIXIMAB ORIGINATOR IN PATIENTS WITH TAKAYASU ARTERITIS WITHIN THE RTU (TEMPORARY RECOMMENDATION OF USE) IN FRANCE}

J.-F. Kleinmann ${ }^{1}$, L. Arnaudd ${ }^{1}$, E. Sauleau ${ }^{2}$, N. Gouyette ${ }^{3}$, R. Tajima ${ }^{1}$, J. Sibilia ${ }^{1}$ on behalf of CRI (Club Rhumatismes et Inflammation). ${ }^{1}$ Rheumatology; ${ }^{2}$ Public Health, Strasbourg's University Hospital, Strasbourg; ${ }^{3}$ MSD France, Courbevoie, France

Background: The benefit/risk ratio of infliximab in refractory patient with Takayasu arteritis (TA) is assumed to be favorable, based on retrospective studies with limited sample size [1, 2] in which infliximab has been prescribed off-label.

Since 2013, the French Temporary Recommendation of Use (RTU) provides a temporary framework allowing the use of infliximab originator in "TA patients refractory to conventional treatment" during a 3-year period.

Objectives: The aim of the study was to evaluate the real-life efficacy and safety of infliximab originator in TA patients initiating or with ongoing infliximab treatment. 
Methods: Prospective, multicenter, observational, open-label, study of TA patients treated with infliximab originator.

Results: As of 2016 (2 years of follow-up), the study included 14 patients (13/14 were female; median age 32 [range 12-56] years) with TA (diagnosis according to Ishikawa criteria). At study entry, the median TA duration was 4.7 (0.4-16) years, 12 patients were already treated with infliximab originator (for a median duration of 34 [1-78] months) and 11 patients were receiving corticosteroids (CS). Infliximab originator was administered at a median dose of $5(3-6) \mathrm{mg} / \mathrm{kg}$ with a median interval of $8(2-67)$ weeks between each infusion. Seven patients had an improvement in at least 1 of the NIH disease activity criteria for TA [3] after a median follow-up time of $2(0.4-11)$ months), 4 had smoldering disease and 1 patient worsened.

The median daily dose of CS decreased from $13(5-35) \mathrm{mg} /$ day of prednisone or equivalent at baseline to $7(5-10) \mathrm{mg} /$ day at 6 months, to $5(5-6) \mathrm{mg} /$ day at 12 months, and remained stable up to 22 months of follow-up.

Infliximab was discontinued in 6 patients due to inefficacy $(n=3)$, prior to pregnancy $(n=2)$, or remission of TA $(n=1)$. Two adverse events ( 1 disease flare and 1 spontaneous miscarriage) were reported, without infliximab interruption.

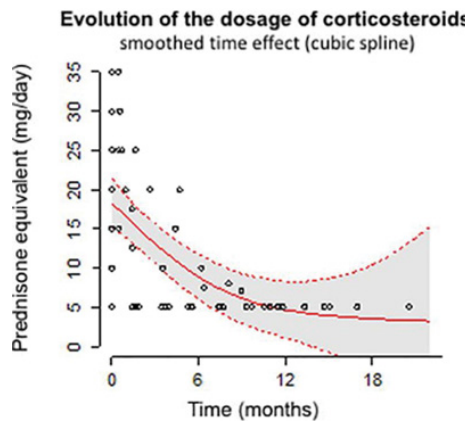

Conclusions: Infliximab originator appears to provide an effective and favourable benefit:risk treatment option to refractory TA patients.

\section{References:}

[1] Mekinian et al, Efficacy of Biological-Targeted Treatments in Takayasu Arteritis: Multicenter, Retrospective Study of 49 Patients, Circulation 132(18) (2015) 1693-700.

[2] Mekinian et al, Efficacy and tolerance of infliximab in refractory Takayasu arteritis: French multicentre study, Rheumatology (Oxford) 51(5) (2012) 882-6. [3] Kerr and al. Takayasu arteritis. Ann Intern Med. 1994; 120: 919-29.

Acknowledgements: The patients were included by AP-HP Cochin ( $\mathrm{Dr} X$ Puéchal), CHU Angers (Dr C Lavigne), CHU Tours (Pr E Diot), CHU Besançon ( $\mathrm{Pr}$ N Magy, Dr H Gil), CHU Lille (Pr E Hachulla, Pr M Lambert, Dr H Maillard), AP-HP Kremlin-Bicêtre (Pr I Koné-Paut), AP-HM Marseille (Pr JR Harlé, Dr N Schleinitz), HEGP Pompidou (Dr JE Kahn), CH Royan (Dr A Bonnin), CHU Strasbourg (Pr T Martin, Dr V Poindron), CHU Bordeaux (Pr J Constans).

Disclosure of Interest: J.-F. Kleinmann Consultant for: MSD, L. Arnaud: None declared, E. Sauleau Consultant for: MSD, N. Gouyette Employee of: MSD, R. Tajima: None declared, J. Sibilia Consultant for: MSD

DOI: 10.1136/annrheumdis-2017-eular.1793

\section{FRI0330 FATIGUE AND DEPRESSION IN PATIENTS WITH BEHCET'S DISEASE}

J.L. Silva, D. Santos-Faria, J. Sousa-Neves, J. Rodrigues, J. Costa, C. Afonso D. Peixoto. Rheumatology, ULSAM, Ponte de Lima, Portugal

Background: Behcet's disease (BD) is a systemic vasculitis of unknown etiology defined by a classic triad consisting of aphtous ulcers of the mouth and genital and uveitis. In addition to these, BD may involve cardiovascular, pulmonar, neurological, articular and gastrointestinal manifestations. Fatigue, anxiety and depression are important problems in chronic inflammatory diseases. There are few studies on fatigue in BD patients. Ilhan et al. ${ }^{1}$ reported more fatigue in BD patients than in healthy controls.

Objectives: The objective of this study was to investigate fatigue and depression in patients with BD and to examine the relationship between these symptoms and disease activity and gender.

Methods: Forty four patients with $\mathrm{BD}$ and thirty nine healthy controls $(\mathrm{HC})$ were included in this study. Age, gender, disease duration and clinical involvements were recorded. All subjects completed the FACIT Fatigue Scale (FACIT-F), Short Form-36 (SF-36), Hospital Anxiety and Depression Scale (HADS) and Health Assessment Questionnaire (HAQ). Disease activity among BD patients was assessed using the Birmingham Vasculitis Activity Score (BVAS)

Results: The mean age was 40.0 years for BD patients and 38.7 for $\mathrm{HC}$. $77 \%$ of $\mathrm{BD}$ patients were women. The mean disease duration for BD patients was 6.1 years. All patients had oral ulcers and genital ulceration; 20 had pseudofolliculitis; 9 had uveitis, 9 had erythema nodosum, 12 had articular involvement, 5 patients had vascular involvement, 4 had pathergia, 1 had orchitis and one had gastrointestinal involvement. Thirty two BD patients were taking medication, including colchicine or DMARDs or their combinations. Seventeen patients had inactive disease and twenty seven had BVAS $\geq 1$. As shown in Table 1, both the HADS-depression (HADS-D) (4.7 vs 2.5) and HADS-anxiety (HADS-A) (8.3 vs 5.7) scores were elevated in $\mathrm{BD}$ patients compared to healthy controls. FACIT-F was higher in healthy controls in comparison with BD group (44.1 vs 36.6), revealing lower levels of fatigue. There were no differences between gender on these scores.

Table 1. Comparison of BD patients and $\mathrm{HC}$

\begin{tabular}{lccc}
\hline Scales & $\mathrm{BD}(\mathrm{n}=44)$ & $\mathrm{HC}(\mathrm{n}=39)$ & P-value \\
\hline FACIT-F & $36.6 \pm 7.3$ & $44.1 \pm 6.4$ & $<0.001$ \\
HADS-D & $4.7 \pm 2.4$ & $2.5 \pm 2.4$ & $\mathbf{0 . 0 0 4}$ \\
HADS-A & $8.3 \pm 4.2$ & $5.7 \pm 2.8$ & $\mathbf{0 . 0 3}$ \\
\hline
\end{tabular}

There were no significant differences in FACIT-F, HADS-A and HADS-D scores with the disease activity $(P=0.952, P=0.391$ and $P=0.286$, respectively).

FACIT-F had a negative correlation with $\mathrm{HAQ}$ and HADS and a positive correlation with the different components of SF-36 and age. FACIT-F score was not correlated with disease duration (Table 2).

Table 2. Correlations between FACIT-F and HAQ, HADS-A, HADS-D, SF-36, age, disease duration in $\mathrm{BD}$

\begin{tabular}{lcccccc}
\hline & HAQ & HADS-A & HADS-D & SF-36 & Age & Disease duration \\
\hline r-value & -0.670 & -0.569 & -0.562 & 0.857 & -0.370 & 0.053 \\
P-value & $<\mathbf{0 . 0 0 1}$ & $<\mathbf{0 . 0 0 1}$ & $\mathbf{< 0 . 0 0 1}$ & $\mathbf{< 0 . 0 0 1}$ & $\mathbf{0 . 0 1 3}$ & $\mathbf{0 . 1 0 3}$ \\
\hline
\end{tabular}

Conclusions: In our study, fatigue and increased levels of anxiety and depression were more common in BD patients. Contrary to the study of Ilhan et al ${ }^{1}$, we found that fatigue was not higher in patients with active disease. Similarly, there were no correlation between the assessed scores and gender. In spite of these results, the authors believe that controlling the symptoms may improve the quality of life in BD patients.

References:

[1] Ilhan et al. Fatigue in patients with Behcet's syndrome: relationship with quality of life, depression, anxiety, diability and disease activity. International Journal of Rheumatic Diseases. 2016.

Disclosure of Interest: None declared

DOI: 10.1136/annrheumdis-2017-eular.4459

\section{FRI0331 IDENTIFICATION AND VALIDATION OF POTENTIAL METABOLOMIC BIOMARKERS FOR RELIABLE DIAGNOSIS OF BEHCET'S DISEASE USING GAS CHROMATOGRAPHY WITH TIME-OF-FLIGHT MASS SPECTROMETRY}

J.K. Ahn ${ }^{1}$, J. Hwang ${ }^{2}$, H.-S. Cha ${ }^{3}$, E.-M. Koh ${ }^{3} .{ }^{1}$ Department of Internal Medicine, Kangbuk Samsung Hospital, Sungkyunkwan University School of Medicine; ${ }^{2}$ Department of Internal Medicine, Natonal Police Hospital;

${ }^{3}$ Department of Medicine, Samsung Medical Center, Sungkyunkwan University School of Medicine, Seoul, Korea, Republic Of

Background: Although many diagnostic criteria have been developed and revised by experts in the field, diagnosing Behcet's disease (BD) is still complicated and challenging. Metabolomic studies can lead to a better understanding of pathophysiological processes and may uncover new diagnostic markers for classification of disease subgroups and assessment of disease activity in rheumatic diseases. No metabolomic studies on serum have been attempted to improve the diagnosis and to identify potential biomarkers of BD.

Objectives: The purposes of this study were to investigate distinctive metabolic changes in serum samples of BD patients and to identify metabolic candidate biomarkers for reliable diagnosis of BD using the metabolomics platform.

Methods: Metabolomic profiling of 90 serum samples from 45 BD patients and 45 healthy controls $(\mathrm{HC})$ was performed via gas chromatography with time-of-flight mass spectrometry (GC/TOF-MS) with multivariate statistical analyses. Thirty-five patients with $\mathrm{BD}$ and age- and sex-matched $35 \mathrm{HCs}$ were randomly selected to form the discovery set, and the validation set is composed of 10 patients with BD and 10 HCs.

Results: We identified a total of 104 metabolites in the serum samples of the discovery set. To maximise the discrimination between groups $\mathrm{BD}$ and $\mathrm{HCs}$, we applied supervised partial least squared-discrimination analysis (PLS-DA). A PLS-DA score plot of the discovery set indicated that the cluster of BD patients is well separated from $\mathrm{HC}$ clusters in component 1 . The variation values of the PLS-DA model are $R^{2} X$ of $0.246, R^{2} Y$ of 0.913 , and $Q^{2}$ of 0.852 , respectively, indicating strong explanation and prediction capabilities of the PLS-DA model. The PLS-DA models did not show clear and consistent discrimination between groups according to drug administration, indicating that drugs used in patients with $\mathrm{BD}$ had a negligible effect on the distinct metabolic profiles discriminating BD patients from HCs. A panel of five metabolic biomarkers, namely, decanoic acid, fructose, tagatose, linoleic acid, and oleic acid were selected based on their variable importance on projection values and adequately validated as putative biomarkers of BD (sensitivity $100 \%$, specificity $97.1 \%$, area under the curve 0.993 ) in the discovery set and validation set. Principal component analysis showed clear discrimination of $\mathrm{BD}$ and $\mathrm{HC}$ groups by the five metabolic biomarkers in the validation set.

Conclusions: This is the first report on detection of a characteristic metabolic profile of $\mathrm{BD}$ and identifying and validating potential metabolite biomarkers in 